Document downloaded from:

http://hdl.handle.net/10251/157215

This paper must be cited as:

Torres, V.; Sánchez, N.; Etayo, D.; Ortuño, R.; Martínez Abietar, AJ.; Navarro-Cía, M.; Beruete, M. (2014). Extraordinary Transmission-inspired Dual-band THz Quarter-wave Plate. OSA. 1-2. https://doi.org/10.1364/CLEO_SI.2014.STh3F.8

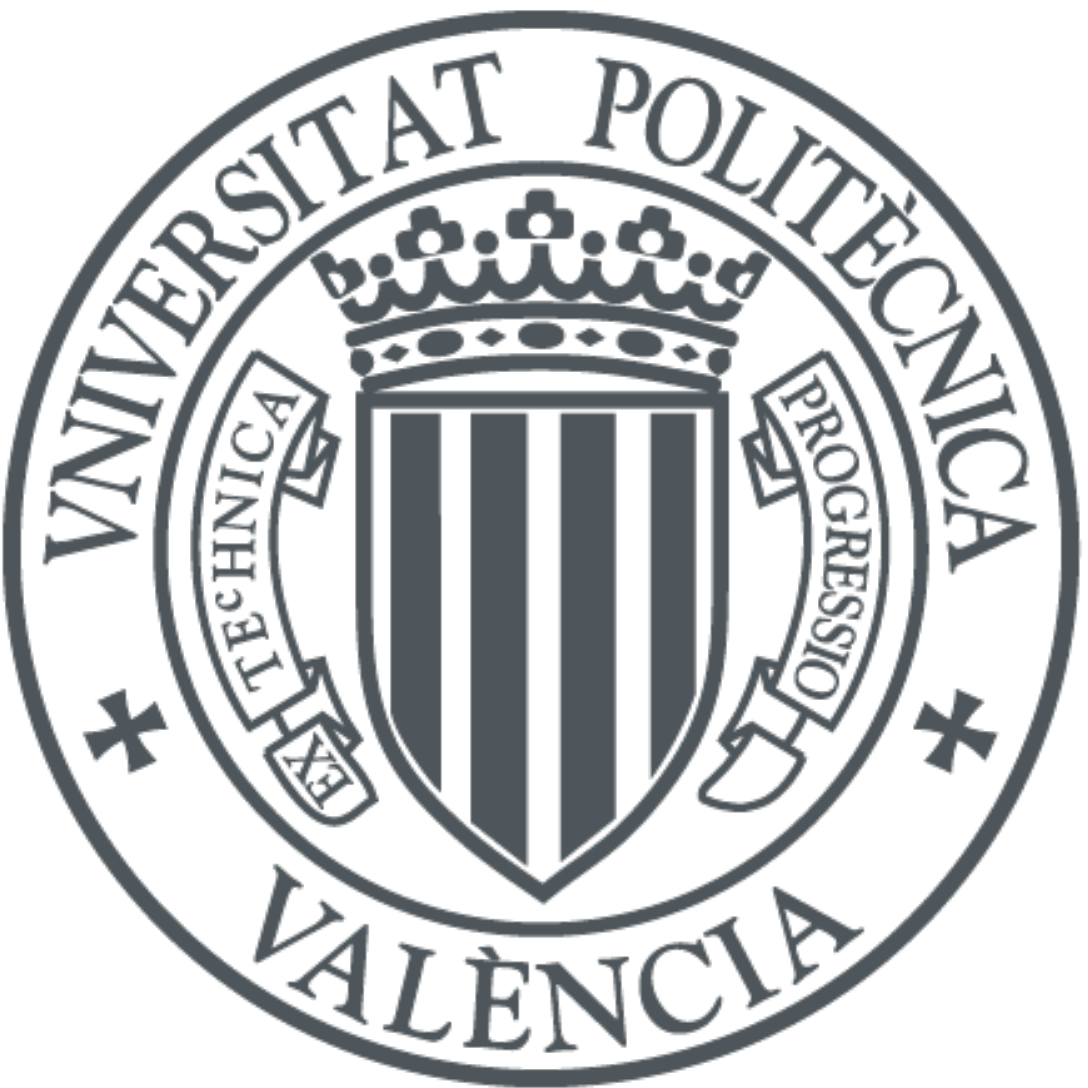

The final publication is available at

https://doi.org/10.1364/CLEO_SI.2014.STh3F.8

Copyright OSA

Additional Information 


\title{
Extraordinary Transmission-inspired Dual-band THz Quarter-wave Plate
}

\author{
Víctor Torres ${ }^{1}$, Nuria Sánchez ${ }^{2}$, David Etayo ${ }^{3}$, Rubén Ortuño ${ }^{1}$, Alejandro Martínez ${ }^{2}$, Miguel Navarro-Cía ${ }^{4,5}$, and \\ Miguel Beruete ${ }^{1}$ \\ ${ }^{1}$ TERALAB (MmW - THz - IR \& Plasmonics Laboratory), Universidad Pública de Navarra, Pamplona 31006, Spain \\ ${ }^{2}$ Nanophotonics Technology Center, Universitat Politècnica de València, 46022, Valencia, Spain \\ ${ }^{3}$ Antenna Group, Electric and Electronic Department, Universidad Pública de Navarra, Pamplona 31006, Spain \\ ${ }^{4}$ Department of Electrical and Electronic Engineering, Imperial College London, South Kensington Campus, London SW7 2BT, UK \\ ${ }^{5}$ Department of Electronic \& Electrical Engineering, University College London, Torrington Place, London WC1E 7JE, UK \\ m.navarro@imperial.ac.uk
}

\begin{abstract}
We propose a very compact metasurface that works as a quarter-wave plate at two different frequencies, $1 \mathrm{THz}$ and $2.2 \mathrm{THz}$. The fractional bandwidth of the first band is remarkably $32.2 \%$, beyond the state-of-the-art.

OCIS codes: (050.2555) Form birefringence; (240.6690) Surface waves; (300.6495) Spectroscopy, terahertz;
\end{abstract}

\section{Introduction}

An unprecedented activity in terahertz is driven by its promising application in a wide range of fields like security surveillance, high-throughput wireless transmission, semiconductor characterization and medical imaging [1]. To satisfy the high performance demands, a new generation of quasi-optical devices with additional functionalities is needed.

Quasi-optical polarizers are unquestionably of great interest for science and technology. Classical polarizers benefit from the birefringence properties of natural materials [2]. However, they are bulky. Hence, researchers proposed to generate artificially such birefringence with periodic structures [3-6]. Unfortunately, the reduction in size came at expense of bandwidth of operation. For continuous-wave sources this is not a disadvantage. Nevertheless, broadband time-domain sources are playing an important role in revitalizing the THz-gap. Therefore, researchers need to engineer compact polarizers (e.g. quarter-wave plates) with enhanced operation bandwidths.

We address here the problem by exploiting an extraordinary transmission metasurface. In the past we proposed a narrowband quarter-wave plate with similar metasurfaces [7]. Here, such metasurface is modified slightly to keep such narrowband operation and to display an additional band at lower frequencies where the metasurface is offresonance. As a result of the off-resonance situation, the fractional band of such fundamental band surpasses that of single-layer frequency-selective-surface based quarter-wave plates reported in the literature.

\section{Fundamentals, setup and numerical \& experimental results}

As it is shown in Fig. 1(a), where a scanning electron microscopy photograph of the prototype is presented together with the structural parameters, the metasurface is a modified extraordinary transmission hole array: holes are connected by vertical slits [8] and their lateral sides are meander-lines [9]. The structural parameters of the unit cell are the following: $d_{y}=120 \mathrm{\square m}, d_{x}=58 \mathrm{\square m}$, slit width $w=4.5 \mathrm{Dm}$, hole size $a_{x}=41.3 \mathrm{Dm}$ and $a_{y}=47.9 \mathrm{Dm}$, meander width $m=4.5 \mathrm{Dm}$ and meander separation $\mathrm{s}=7.475 \mathrm{Dm}$. The metal is gold with a thickness of $0.1 \mathrm{Dm}$ and modeled with a constant electric conductivity equal to $4.561 \times 10^{7} \mathrm{~S} / \mathrm{m}$. All the structure is backed by a substrate of polypropylene of permittivity $\square_{\mathrm{r}}=2.2201$, loss tangent $\tan \mathrm{CD}=0.001$ and thickness $t=40 \mathrm{um}$. The fabrication process is based on wellknown "lift-off process", widely used in silicon photonics, adapted here to work with flexible substrates instead of silicon based ones.

The finite-integration-based software CST Microwave Studio ${ }^{\mathrm{TM}}$ is used to optimize the unit cell parameters within the fabrication constraints to achieve quarter-wave plate behavior at two frequencies. The experiment was performed using a TPS Spectra 3000 from Teraview under nitrogen purging condition. This equipment is a floorstanding terahertz transmission time domain spectrometer for the frequency range 0.060-3.5 THz.

Experimental and numerical results for the two fabricated prototypes are shown in Fig. 1(b). From a circuit point of view, the behavior of the prototype can be explained easily. Under $x$-polarization (horizontal electric field), the electromagnetic wave effectively sees a slit array which behaves as a capacitance [10]. Hence, the transmission tends to one at low frequency. On the other hand, under $y$-polarization (vertical electric field), the wave sees a hole array which is known to have an inductive behavior at low frequencies [11] and therefore, the transmission tends to zero at DC. This performance, based on complementary capacitive and inductive response, allows controlling the phase retardation between the orthogonal components at the first band $(\sim 1 \mathrm{THz})$. Since at this first band the 
operation is off-resonance, an unprecedented large fractional bandwidth is realizable. The second band ( 2.2 THz) is achieved however by exploiting the Fabry-Perot resonances within the dielectric substrate. Given the resonant character, the fraction bandwidth at this band is below 5\%, in accordance with state-of-the-art resonantfrequencyselective-surface-based quarter-wave plates. Notice that the peak at $\sim 1.5 \mathrm{THz}$ for $E_{y}$-polarized wave is the extraordinary transmission peal. At this frequency, the locally-induced higher order mode $\mathrm{TM}_{02}$ compensates the inductive behavior of the hole array and leads to a high transmittance peak.

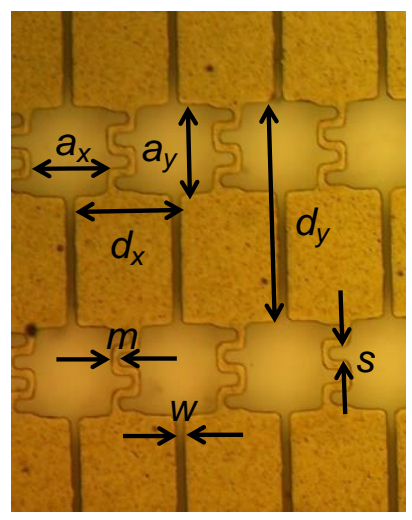

(a)

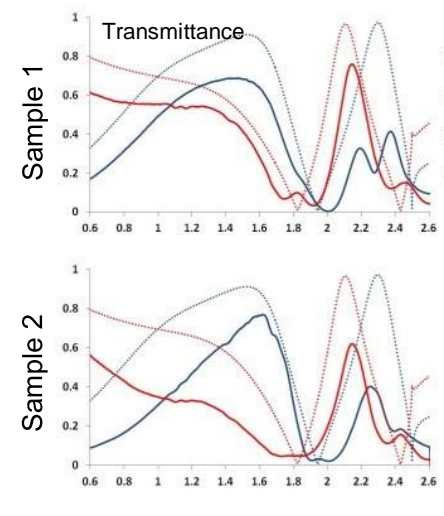

Fig. 1. (a) SEM photograph of sample 1 together with unit cell parameters. (b) From left to right: amplitude transmittance for $E_{x}$ (red curves) and $E_{y}$ (blue curves), amplitude and phase difference for orthogonal components. Solid lines represent experimental results and dotted lines simulation ones.

\section{Conclusions}

In conclusion, a polarization device which converts the polarization from linear to circular (and vice versa) at two different bands in the THz-gap is presented. The birefringence is achieved by means of the excitation of a complementary capacitive and inductive response which is obtained through a single layer extraordinary transmission square hole array backed with a polypropylene substrate, where the holes are connected with slits and meander lines along the vertical and horizontal direction, respectively. Wide and narrow operation bandwidths of $32.2 \%$ and $2.3 \%$ at $1 \mathrm{THz}$ and $2.2 \mathrm{THz}$, respectively, are obtained after numerical analysis. Moreover, prototypes have been fabricated and measured demonstrating a similar performance.

\section{Acknowledgements}

This work was supported in part by the Spanish Government under Contract Consolider Engineering Metamaterials CSD2008-00066 and contract TEC2011-28664-C02. M. Navarro-Cía acknowledges his IC Junior Research Fellowship.

\section{References}

[1] Y.-S. Lee, Principles of Terahertz Science and Technology, New York: Springer, 2009.

[2] C. Jördens, M. Scheller, M. Wichmann, M. Mikulics, K. Wiesauer, and M. Koch, "Terahertz birefringence for orientation analysis," Appl.

Opt. 48, 2037 (2009).

[3] J.-B. Masson and G. Gallot, "Terahertz achromatic quarter-wave plate," Opt. Lett. 31, 265 (2006).

[4] B. Scherger, M. Scheller, N. Vieweg, S. T. Cundiff, and M. Koch, "Paper terahertz wave plates," Opt. Express 19, 24884-24889 (2011).

[5] S. C. Saha, Y. Ma, J. P. Grant, A. Khalid, and D. R. S. Cumming, "Low-Loss Terahertz Artificial Dielectric Birefringent Quarter-Wave Plates," IEEE Photonics Technol. Lett. 22, 79-81 (2010).

[6] A. C. Strikwerda, K. Fan, H. Tao, D. V. Pilon, X. Zhang, and R. D. Averitt, "Comparison of birefringent electric split-ring resonator and meanderline structures as quarter-wave plates at terahertz frequencies," Opt. Express 17, 136-149 (2008).

[7] M. Navarro-Cía, P. Rodriguez-Ulibarri, V. Torres, and M. Beruete, "Quarter-Wave Plate Based on Dielectric-Enabled Extraordinary Resonant Transmission," IEEE Photonics Technol. Lett. 24, 945-947 (2012).

[8] M. Beruete, M. Sorolla, M. Navarro-Cía, and I. Campillo, "Polarized left-handed extraordinary optical transmission of subterahertz waves," Opt. Express 15, 8125-8134 (2007).

[9] M. Beruete, M. Navarro-Cía, V. Torres, and M. Sorolla, "Redshifting extraordinary transmission by simple inductance addition," Phys. Rev.

B 84, 075140-1-5 (2011).

[10] N. Marcuvitz, in Waveguide Handbook, London, UK: McGraw-Hill, 1951.

[11] F. Medina, F. Mesa, and R. Marqués, "Extraordinary Transmission Through Arrays of Electrically Small Holes From a Circuit Theory Perspective," IEEE Trans. Microw. Theory Tech. 56, 3108-3120 (2008). 